\title{
Iatrogenic Injury of Right Coronary Artery During Tricuspid Annuloplasty
}

Satoshi Kainuma, MD, PhD; Hiroya Yamamoto, MD; Shohei Yamada, MD;

Yu Yamada, MD; Akinori Sumiyoshi, MD; Atsunori Okamura, MD, PhD;

Katsuomi Iwakura, MD, PhD; Yuka Miyata, MD, PhD; Yukio Hayashi, MD, PhD;

Masakazu Ueda; Yoshiki Sawa, MD, PhD; Takafumi Masai, MD, PhD

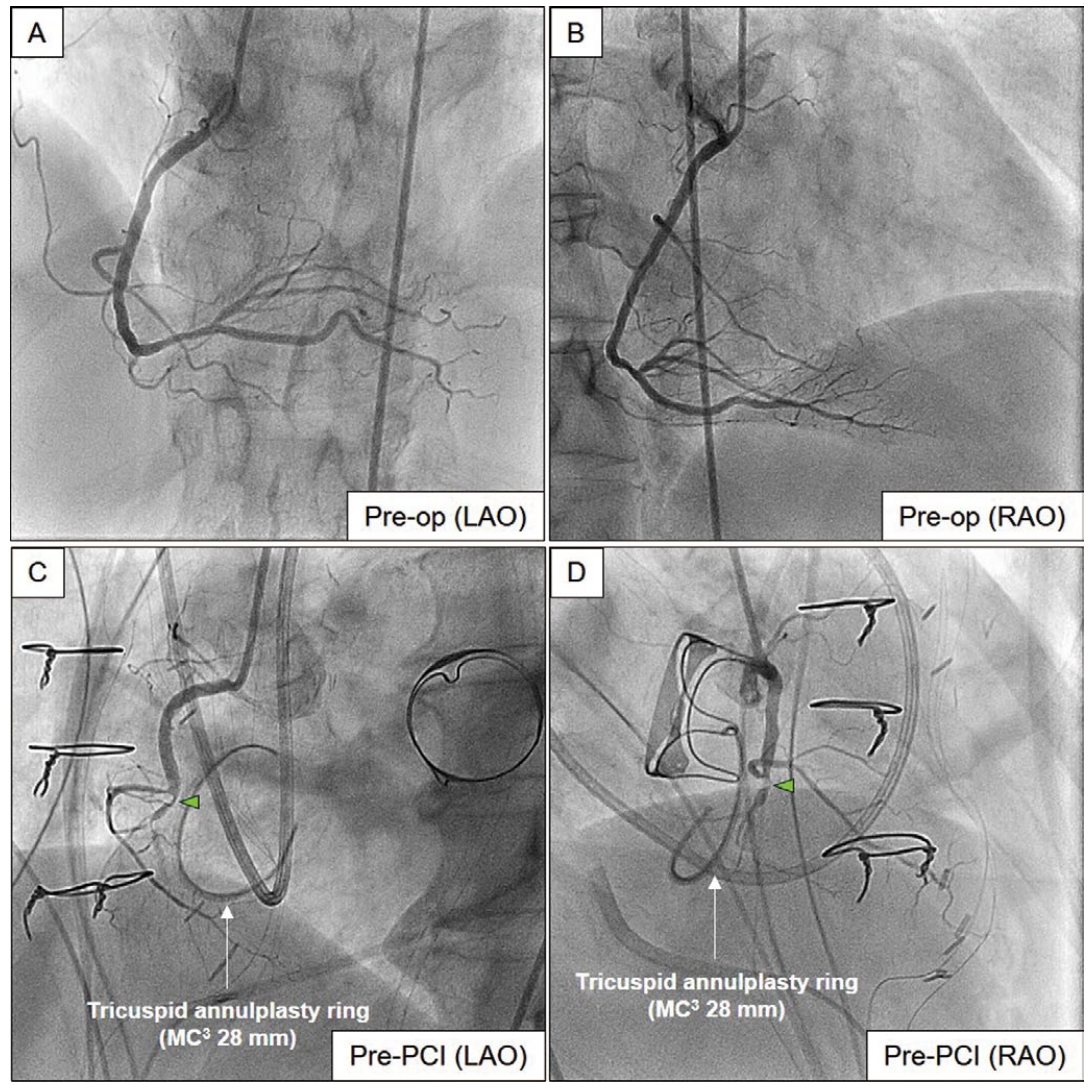

Figure 1. (A,B) Preoperative angiography showing the intact right coronary artery. (C,D) Postoperative angiography showing occlusion of the right coronary artery just distal to the right marginal artery. $L A O$, left anterior oblique; RAO, right anterior oblique.

A 71-year-old woman with refractory heart failure resulting from rheumatic mitral valve disease, severe tricuspid regurgitation, and atrial fibrillation was referred for surgery. Preoperative coronary arteriography showed a stenotic lesion in the proximal segment of the left ascending coronary artery and an intact right coronary artery (Figure 1A,B).

Under a standard cardiopulmonary bypass approach with intermittent cold blood cardioplegia, left sided maze procedure and mitral valve replacement with a $25-\mathrm{mm}$ Magna Mitral Ease Heart Valve (Edwards Lifesciences, Irvine, CA, USA) were performed. Snares were placed

Received February 15, 2017; revised manuscript received March 23, 2017; accepted April 3, 2017; released online April 22, 2017 Time for primary review: 17 days

Department of Cardiovascular Surgery (S.K., H.Y., S.Y., Y.Y., T.M.), Department of Cardiology (A.S., A.O., K.I.), Department of Anesthesiology (Y.M., Y.H.), Department of Section of Clinical Echocardiography (M.U.), Sakurabashi Watanabe Hospital, Osaka; Department of Cardiovascular Surgery, Osaka University Graduate School of Medicine, Suita, Osaka (Y.S.), Japan

Mailing address: Satoshi Kainuma, MD, PhD, Department of Cardiovascular Surgery, Sakurabashi Watanabe Hospital, 2-4-32 Umeda, Kita-ku, Osaka 530-0001, Japan. E-mail: s.kainuma19780320@gmail.com

ISSN-1346-9843 All rights are reserved to the Japanese Circulation Society. For permissions, please e-mail: cj@j-circ.or.jp 


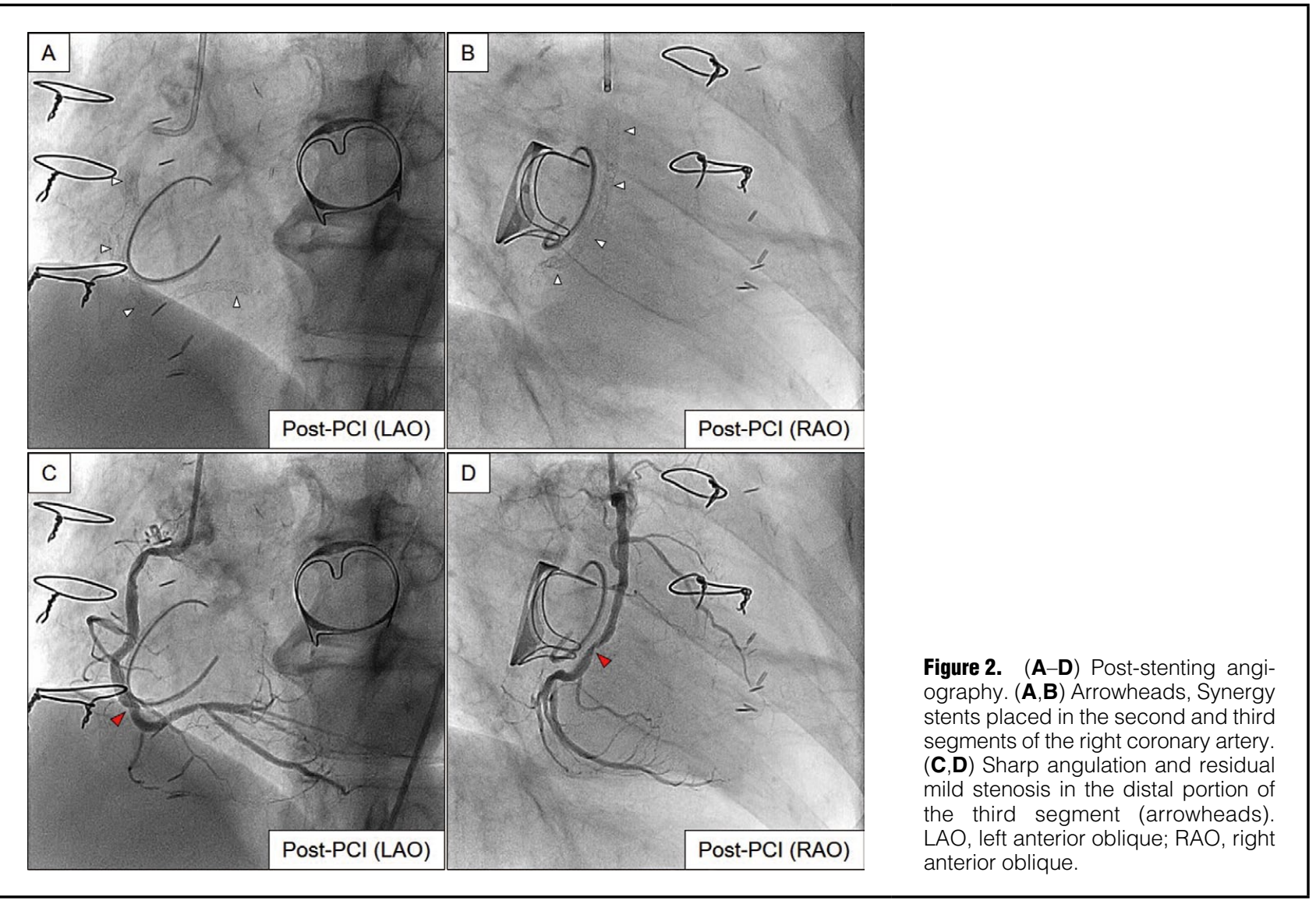

around the inferior and superior vena cava and tightened, then a retrograde cardioplegia catheter was removed at the point on the right atrium after starting a longitudinal incision. Following exposure of the tricuspid valve with a retractor, a right-sided maze procedure was performed. The tricuspid annulus was found to be remarkably dilated, while there was no obvious leaflet abnormality, consistent with secondary tricuspid regurgitation. A total of $10(6$ anterior, 2 posterior, 2 septal annulus, respectively) interrupted horizontal mattress sutures were placed around the tricuspid annulus. The first suture was started from the anteroseptal commissure and run toward the posteroseptal commissure in a clockwise fashion, ending in the middle of the septal leaflet. These sutures were evenly passed through the sewing band of a 28-mm Edwards MC3 Tricuspid Annuloplasty Ring (Edwards Lifesciences), which was lowered down into an annular position and then sutures were sucurely tied. The atriotomy was closed with 4-0 polypropylene with a horizontal mattress running suture, followed by a simple running suture. After sufficient deairing, the aortic cross-clamp was released. Thereafter, coronary artery bypass grafting was performed with the in situ left internal thoracic artery to the distal part of the left ascending coronary artery. The cardiopulmonary bypass was weaned uneventfully and a favorable flow pattern of bypass graft was confirmed with stable hemodynamics. Soon thereafter, however, the patient unexpectedly experienced 2 episodes of ventricular fibrillation, which were initially controlled with direct cardioversion and antiarrhythmic drugs. There were no apparent ST changes and transesophageal echocardiogram showed normal left and right ventricular function, and favorable mitral valve prosthesis function. Several hours after leaving the operating room, the patient developed low cardiac output syndrome refractory to intensive drug support with vasoactive and inotropic agents. Although urgent echocardiography showed normal prosthetic valve function, severe hypokinetic wall motion of the inferior left ventricle was also shown. In addition, a substantial increase in cardiac enzymes was noted on laboratory results, indicating the possibility of ongoing myocardial ischemia.

Emergency coronary angiography showed sharp angulation of the proximal part of the right coronary artery followed by occlusion of the second segment just distal to the acute marginal branch (Figure 1C,D). The proximal origin of the occlusive site was found to have a flattened shape on intravascular ultrasound, indicating mechanical retraction as a possible cause. With great effort we were able to cross the severely tightened lesion with a 0.014 -inch coronary wire, followed by percutaneous coronary angioplasty and implantation of a total of 2 drug-eluting stents in the whole range of the third and second segments in sequential order (Synergy stent, $2.5 \times 38,3.0 \times 28 \mathrm{~mm}$, respectively, Boston Scientific; Figure 2A,B). The excessively tightened lesion, however, did not allow for lasting or sufficient dilatation of the stenosis, resulting in insignificant stenosis in the distal portion of the third segment (Figure 2C,D). On preand post-stenting angiography, the 2 portions of the right coronary artery were consequently retracted by the suture with stenotic lesions located on the opposite side of the 
tricuspid annuloplasty ring (Figure 1C,D; Figure 2A-D). Echocardiogram performed prior to discharge showed mildly impaired left ventricular function with no other remarkable changes.

Few cases of iatrogenic right coronary artery injury related to a tricuspid valve repair have been reported, ${ }^{1}$ though there are several specific aspects to consider regarding this condition. First, an unexceptional coronary injury develops between the right marginal artery and crux of the heart in affected patients, which corresponds to the anteroposterior commissure and posterior leaflet annulus of the tricuspid valve along the right ventricular free wall. This is because this region is where the right coronary artery is most closely related to the tricuspid annulus, with a distance $<5 \mathrm{~mm}$ in $>80 \%$ of patients. ${ }^{2}$ Second, various clinical manifestations may occur, such as electrical instability as well as right ventricular or biventricular dysfunction. The time interval between aortic de-clamp and obvious abnormalities of hemodynamic and electrical conditions may depend on the degree of coronary injury. In the present case, it is likely that incomplete coronary occlusion initially occurred, allowing for stable hemodynamics, while some time was required for the injured region to progress to complete occlusion.

In the present case, post-stenting angiography indicated that the coronary injury was not caused by direct obstruction from the annuloplasty suture, but rather by kinking of an artery as a result of marked reduction in atrioventricular circumference. If the coronary injury had been the result of direct obstruction by the annuloplasty suture or right atriotomy closing suture, then the stenotic coronary lesions would have been located on the same side as those sutures. Díez-Villanueva et al suggested that extremely dilated annuli, which in turn require greater plication, probably have increased risk of right coronary artery injury. ${ }^{1}$ In addition, placement of more superficial stitches (not overly aggressive plication), particularly in the danger zone (anteroposterior commissure), would greatly minimize the risk of this complication. The present experience highlights the importance of comprehensive understanding of the right coronary anatomy in relation to the tricuspid annulus and also indicates the need for inclusion of iatrogenic right coronary injury as part of the differential diagnosis for unreasonable hemodynamic or electrical instability following tricuspid annuloplasty.

\section{Disclosures}

The authors declare no conflict of interest.

\section{References}

1. Díez-Villanueva P, Gutiérrez-Ibañes E, Cuerpo-Caballero GP, Sanz-Ruiz R, Abeytua M, Soriano J, et al. Direct injury to right coronary artery in patients undergoing tricuspid annuloplasty. Ann Thorac Surg 2014; 97: 1300-1305.

2. Al Aloul B, Sigurdsson G, Can I, Li J, Dykoski R, Tholakanahalli M. Proximity of right coronary artery to cavotricuspid isthmus as determined by computed tomography. Pacing Clin Electrophysiol 2010; 33: 1319-1323. 